\section{Soune Haints}

CONCERNING

\section{THE DUODENUM AND THE APPENDIX IN INTESTINAL STASIS."}

\author{
[With Special Plate.]
}

BY ALFRED C. JORDAN, M.D.CAMB. MEDICAL RADIOGRAPHER, GUY'S HOSPITAL AND ROYAL HOSPITAL FOR DISEASES OF THE CHEST.

THE symptoms and signs of intestiual stasis aro very definite and frequently very severe, ${ }^{111} 12$ yet one of the most striking points in connexion with intestinal stasis concerns the position of those medical men who are not familiar wi:h the phenomena of the disease when they are brought in thoir practice face to face with a well-marked case. They are quite unable to make a diagnosis, and they aro compelled to seek refuge in some vague explanation, or to use some equally vague "label," such as "neurasthenia" or "dyspepsia," the latter term being usually preceded by an epithet ("acid," "flatulent," "atonic," or the like). It is not until one or more of the secondary changes has set in, or until one or more of the symptoms or signs has assumed great prominence, that the medical man who is

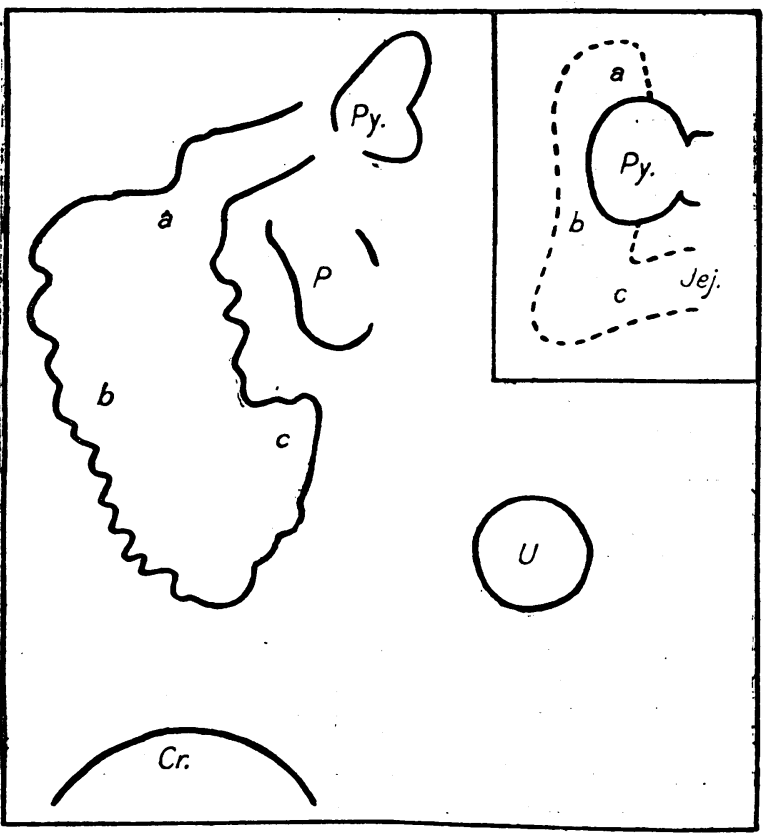

F. 1.-Fluorescent screen tracing (reduced in size) of the duodenum and pylorus in a woman aged 32 , with the typica symptioms and signs of intestinal stasis. Taken on the couch. The duodenum is half as long again and more than double th width of a normal duodenum. For seven or eight minutes the duodenum was observed undergoing vigorous "wriuhing" contractions in a vain endeavour to force its contents in to the jejunum through the kink at the duodeno-jejunal junction. After seven or eich jejum and the coils of the small intestiue. The small figure in the right tended) duodenum drown to the game scale for comporison. distended duodenum of this patient is shown in situ in Fig. 2 , ond an actual skiegram of the duedenum in cause of the duodeno-jejunal kinking is shown in Fig: 4 The patient's main symptom was pain in the region of her duedenum like something distended which would burst if it were tapped upon either in front or behind." $a, b, c$, First, second, and third parts of the duodenum; Py..,pylorus ; $P$, pyloric portion of stomach : $J e j$., jejunum ; $\sigma$, umbilicus; $\boldsymbol{C r}$., crest of ilium.

not familiar with intestinal stasis is able to make a diagnosis. He then diagnoses the secondary conditioncorrectly-but he mistakes it for the primary disease. In other cases one or other of the typical signs of stasis becomes so prominent that it is taken for a disease itself. Thus the staining of the skin may be so pronounced that the patient is thought to be the subject of Addison's disease. An instance of this came to my notice recently; the general lassitude and depression, the poor circulation,

\footnotetext{
* Founded on a demonstration to the Hunterian Society.
}

and the attacks of nausea with loss of flesh lent suppoit to the diagnosis, but the subsiquent course showed it to be erroneous. One of the most constant signs of intestinal stasis in women is a nod $1 \mathrm{ur}$ condition of the breasts, due to chronic mastitis; in $\mathrm{n} 0: \mathrm{c}$ adranced cases the breasts become cystic and ultimately cancerous. A woman was sent into Guy's Hospital a few months ago for amputation of both breasts for supposed malignant disease. They were in a condition of advanced cystic disease. Mr. Arbuthnot Lane sent her to me for $x$-ray examination of the intestines, and I found that there was extreme intestinal stasis. Mr. Lane then treated her case by the operation of "short-circuiting," that is, dividing the ileum near its lower end, and suturing the divided end into the rectum. Within a week after the operation the breasts began to improve, and at the end of three weeks they were practi. cally normal, the only sign of disease now being a small nodule in one breast. The woman's general condition had improved greatly.

\section{The Gall Bladder.}

One result of stasis in the ileum is to allow bacteria to ascend from the large bowel, and to invade the upper reaches of the small intestine. Thus the duodenum is

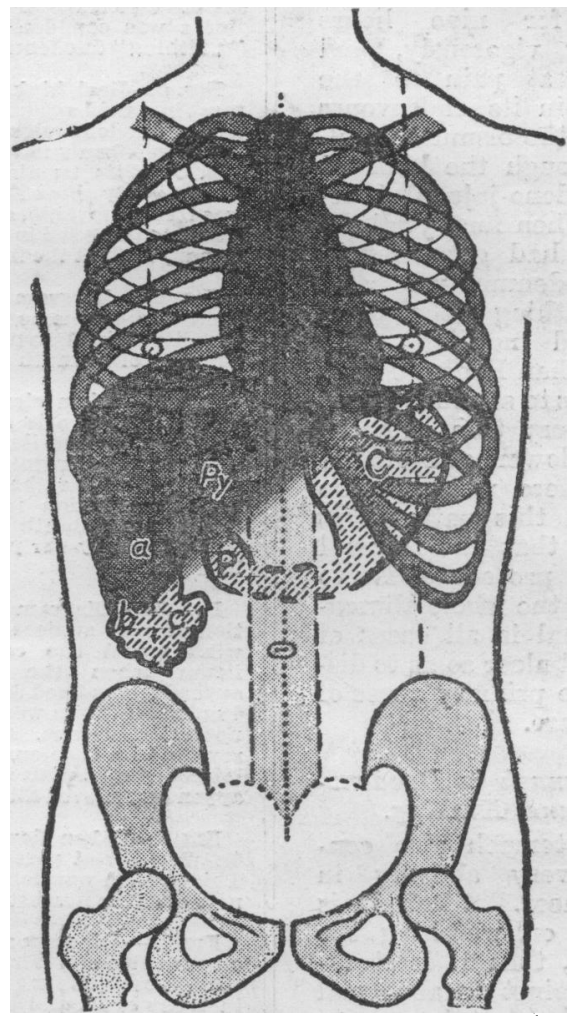

Fig. 2.-Diagram of the stomach and duodenum in the case described in Fig. 1 (and in the text), showing the position they occupied in the body. $a, b, c$, First, second, and third parts of the duodenum; Py., pylorus; C, P, cardiac and pyloric portions

invaded by pathogenic bacteria, which ascend the common bile duct and lead to cholecystitis and chronic pancreatitis. Gall stones are then formed in many cases; if a gall stone finds its way into the bile duct an attack. of acute biliary colic results and is diagnosed, though it is only a secondary result of intestinal stasis.

\section{Duodeno-jejunal Kinking.}

The stasis in the ileum has another effect. The last coils of the ileum are normally placed above the. pelvis; if there be any marked delay in the passage of the ileal contents into the caecum, these last coils of the ileum become overloaded and fall into the pelvis. In falling they drag on the mesentery; this drag is carried to the upper parts of the small intestine, and pulls down the jejunum at its commencement. The third part of the duodenum being (normally) firmly fixed over a peritoneal band, while the jejunum is unsupported at its commencement, a kink is produced at the duodeno-jejunal junction and causes obstruction. The duodenum becomes distended and its 
mucous membrane congested. We have already seen that the duodenum is invaded by pathogenic microbes.; these act on the congested mucous membrane and give rise to duodenal ulceration. "This duodenal kinking has been fully described and illustrated by riè. ${ }^{2}$ I am fréguently able to demonstrate it in patients whose symptoms have led the physician to make the diagnosis (correctly) of duodenal ulcer. I have shown the elongated duodenum, dilated in its first part, the stomach small and of good tone (but sometimes elongated and dropped, though still of good tone), the pylorus perfectly normal, the duodenum contracting actively in repeated ineffectual en. deavours to forward its contents through the kink and into the jejunum: In severe cases the duodenal contractions are very active, amounting to strong writhing movements:

In the most severe case that has come under my notice the duodenum continued for nine hours writhing vigorously, and with great pain to the patient, in its endeavours to force the bismuth emulsion through the kink at the duodeno-jejunal junction. When finally all the bismuth had got through, the duodenum was still seen writhing although it contained nothing more opaque than Benger's food. (Needless to say the patient was a very thin subject.) At the lower end of the ileum there was extreme stasis in this patient. It is clear that the rational line of procedure is to examine the whole alimentary canal in all cases of duodenal ulcer so as to discover the primary cause of the disease.

\section{The Stomach in Duodeno- jejunal Kinking.}

The stomach also contracts very actively in these cases. Often it is evident, on watching the stomach, that it is struggling against an abnormal obstruction. It is equally evident that the obstruction is not at the pylorus, for the bismuth emulsion passes through the pylorus freely. The obstruction is at the duodeno-jejunal junction ; this is absolutely unmistakable : in wellmarked cases

A striking illustration of a case of intestinal stasis, the true nature of which was not understood before the $x$-ray examinations, was brought to my notice recently.

A single woman, aged 32, had suffered from dyspeptic troubles for a year and a half; she had lost a stone in weight during this time. There was loss of appetite and nausea but no vomiting. She complained of pain in the region of the gall bladder. Her "description of the pain was suggestive : "I fee burst if I tap it." The same feeling of a liability of "something distended" to burst if tapped was felt at the corresponding peint at the back. The attacks of pain were intermittent, and were sometimes absent for days. The patient's general condi- tion was very characteristic of intestinal stasis: She was weak and depressed; her hands and feet were cold; the complexion was notice by her rêladitives to be " muddy" in contrast to hër former very clear skin; the breasts showed the nodular condi. tion already described due to chronic mastitis. Two alternative diagnoses had been suggested: Gall stones or a dilated tive diagnoses had been suggested: Gall stones or a dilated
stomach. Renal calculus had also been thought a possible explanation of her symptoms.

I found the stomach elongated and dropped, but normal in other respects. The pylorus was perfectly normal, and the bismuth emulsion passed through the pylorus freely and entered the duodenum. The source of the pain was here encountered, for the duodenum was found to be mueh elongated and dilated to more than double its normal dia meter, except its first part, which appeared to be the seat of cicatricial contrac tion (Fig. 1). The : duodenum was undergoing powerful peristaltic contractions, amounting to strong writhing movements; this continued for seven or eight continued for seven or eight
minutes without a particle of bismuth being able to pass through into the jejunum. The writhing duodenum was observed (and demonstrated to two colleagues) without difficulty, for the duodenum was comfor the duodenum was com-
pletely isolated in the fluorescent screen picture, the rest of the bismuth being contained in the cardiac end of the stomach (Figs. 2 and 3). At the end of seven or eight minutes, with a very powerful duodenal contraction, a large mass of bismuth emulsion was sent suddenly through the duodeno-jejunal junction, and forthwith began to course rapidly through the coils of the jejunum. The subsequent examinations afforded a perfect illustration of the fact (already explained) that the duodenojejunal kinking is secondary to stasis at the lower end of the ileum. After a night's rest, fifteen hours after the bismuth meal, the stomach and duodenum no longer contained any bismuth ; the greater part of it was found to be in the lower coils of the ileum in the pelvis. The caecum also occupied the pelvis and contained a quantity of bismuth, while a small amount was already present in the ascending and transverse colon. Twenty-seven hours after the bismuth meal there was still some bismuth at the lower end .. of the ileum, and the most advanced portion had reached the sigmoid (Fig. 4). Thus the sojourn of the bismuth in the small intestine was more than three times the normal.

The exact cause of the ileal stasis could not be ascertained, for the caecum occupied the deepest part of the pelvis, and it was found impossible to raise the caecum above the pelvic brim, in spite of endeavours in various postures, including the "kneeelbow." Hence the terminal coil of the ileum could not be examined; however this does not detract from the value of the case as an illustration of the fact that the duodenal distension is secondary to the ileal stasis. Then surely no logical person would adrocate the adoption of measures for the relief of the duodenal distension and take no account of the primary disorderthe intestinal stasis. Yet this is the usual procedure at the present time; the duodenal distension is relieved-effectively in most cases-by the operation of gastro- 
DR. A. C. JORDAN: THE DUODENUM AND APPENDIX IN INTESTINAL STASIS.

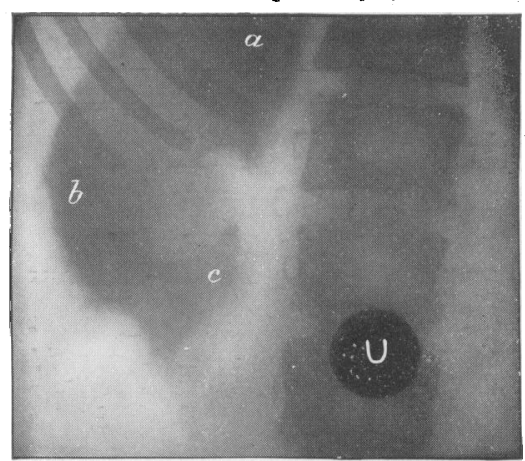

Fig. 3.

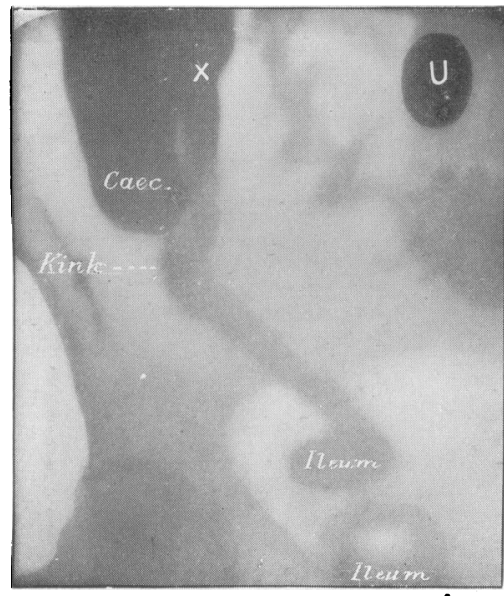

Fig. 5.

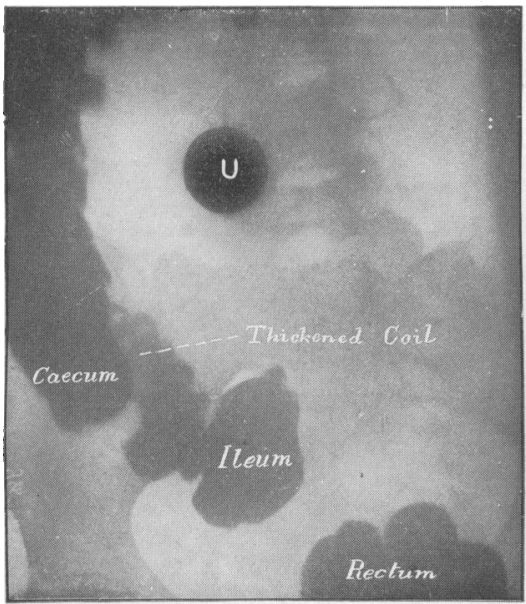

Fig. 6.

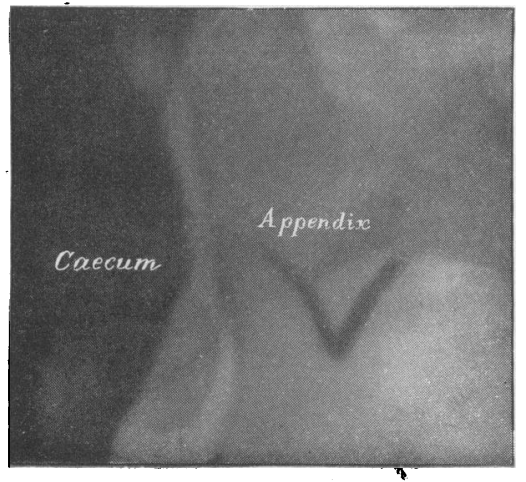

Fig. 7

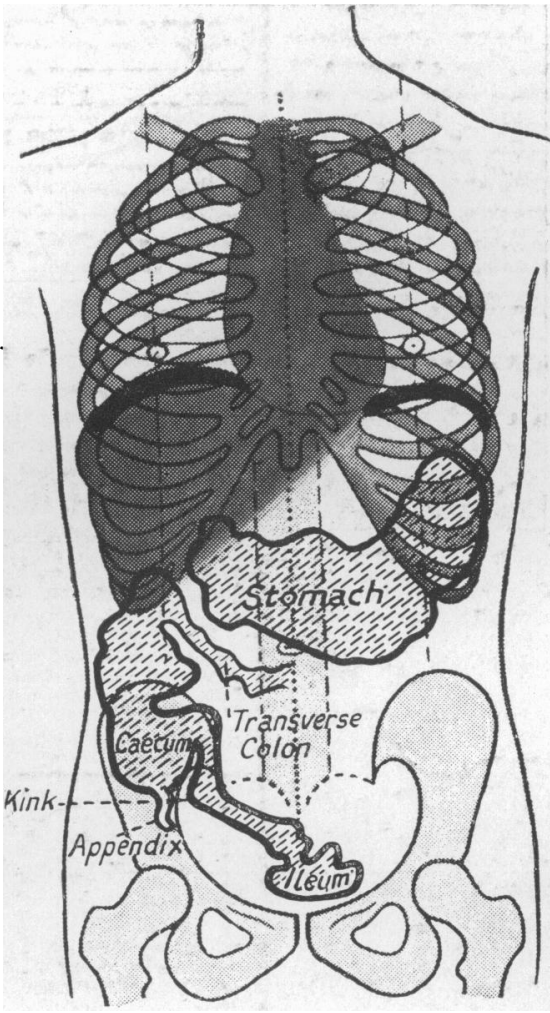

Fig. $5 \mathrm{~A}$.

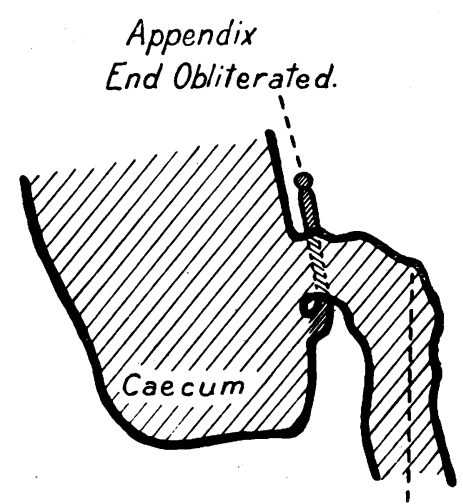

Congested; Irregular. thick-walled lleum. Fig. $6 \mathrm{~A}$.

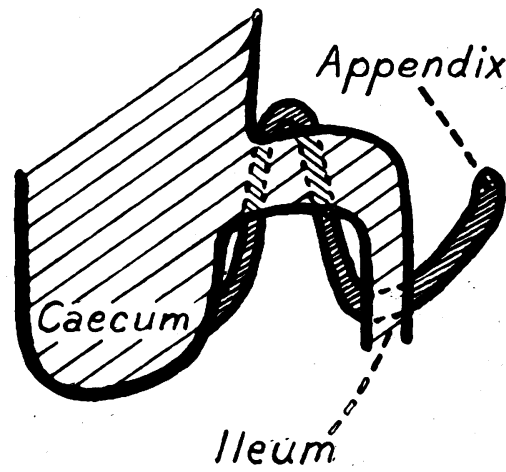

Fig. $7 \mathrm{~A}$

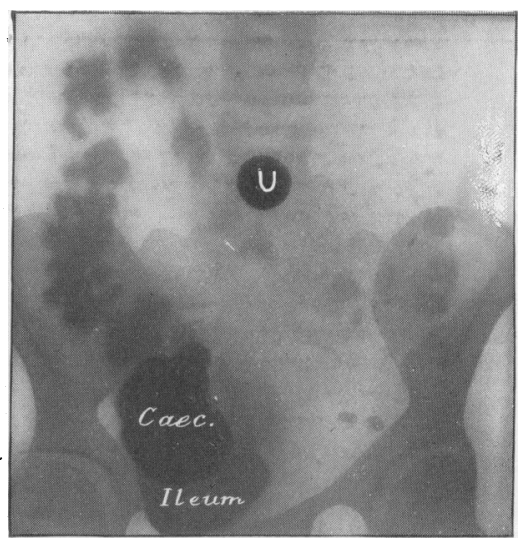

Fig. 4.

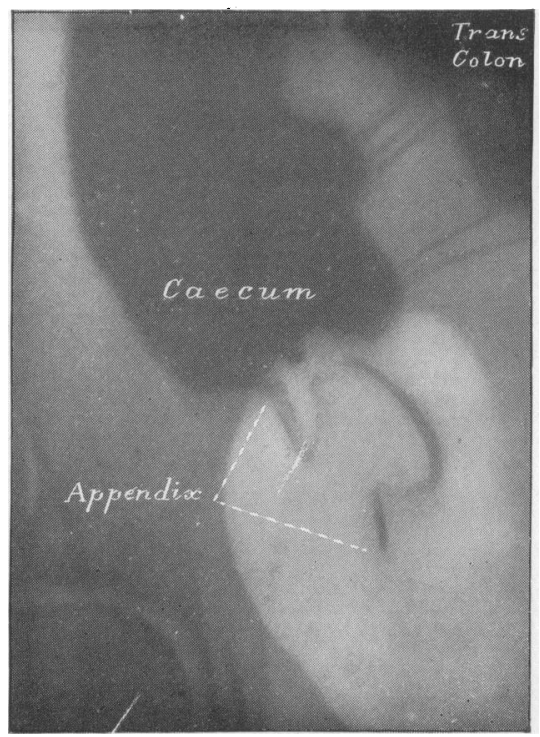

Fig. 8.

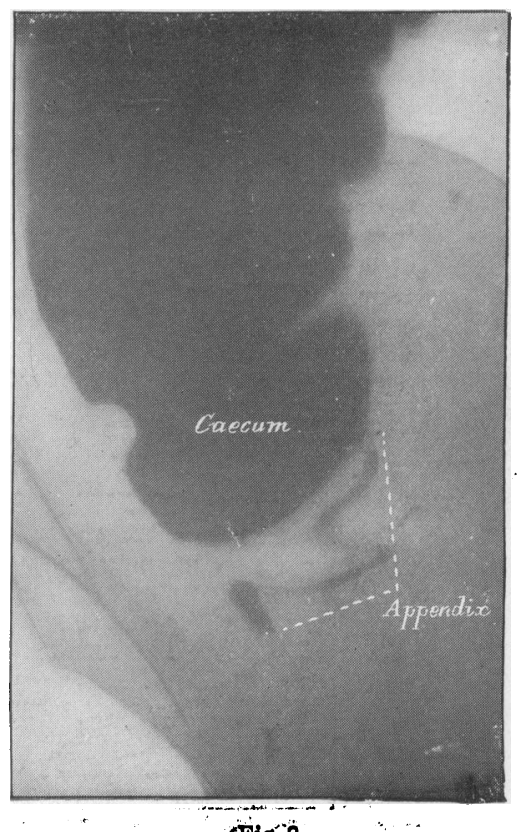

Fig. 9. 
DR. A. C: JORDAN: THE DUODENUM AND APPENDTX IN INTESTINAL STASIS.

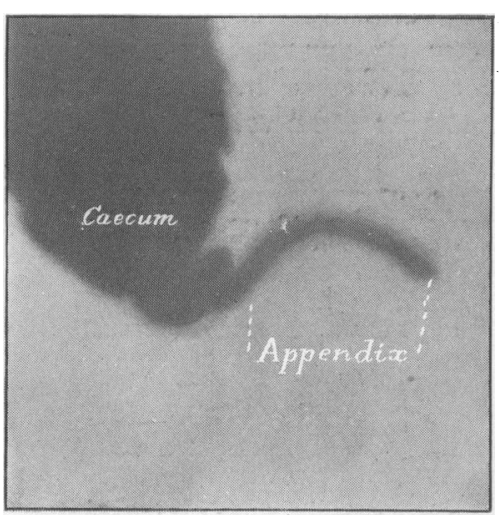

Fig. 10.

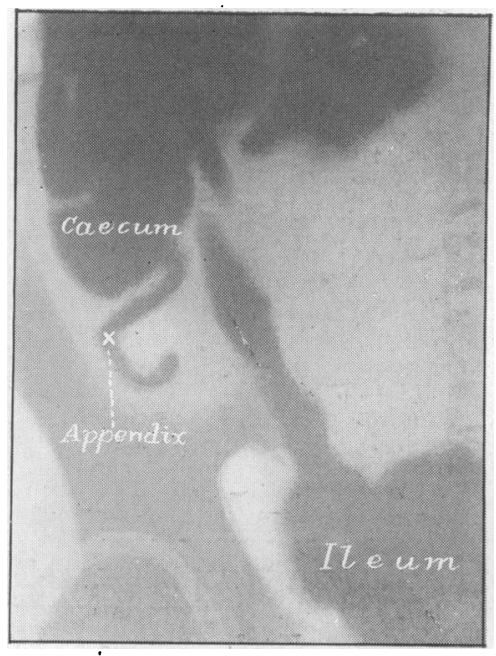

Fig. 13.

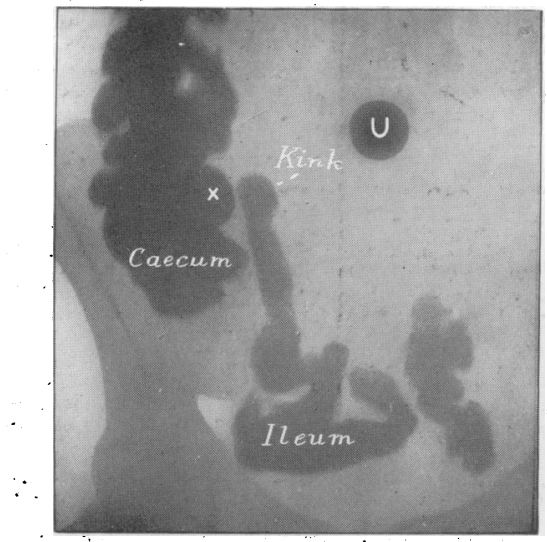

Fig. 11.

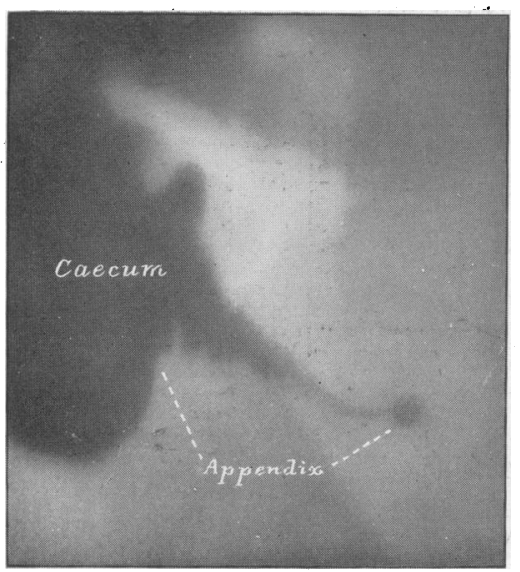

Fig. 14.

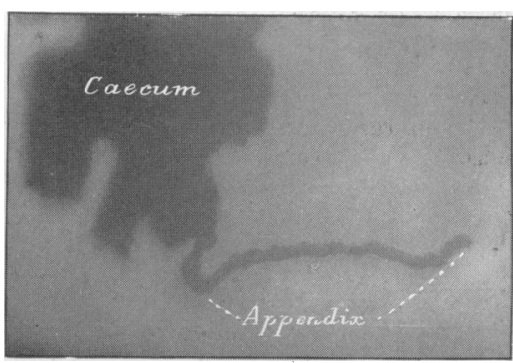

Fig. 12.

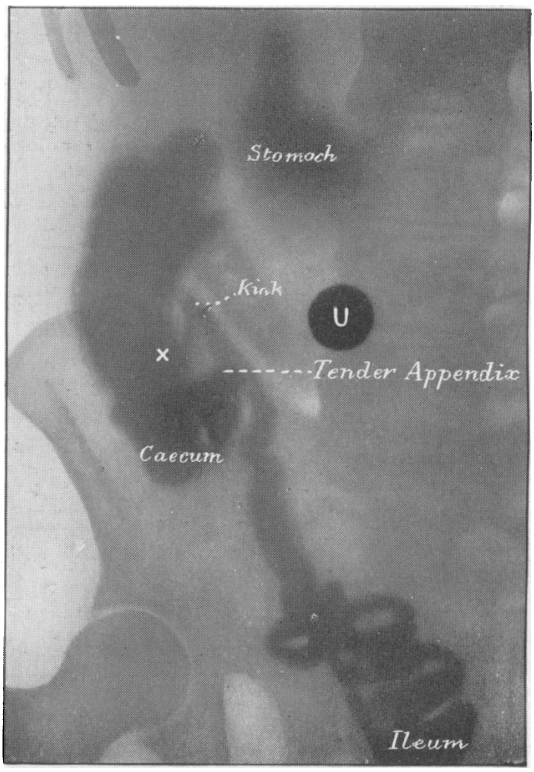

Fig. 15.

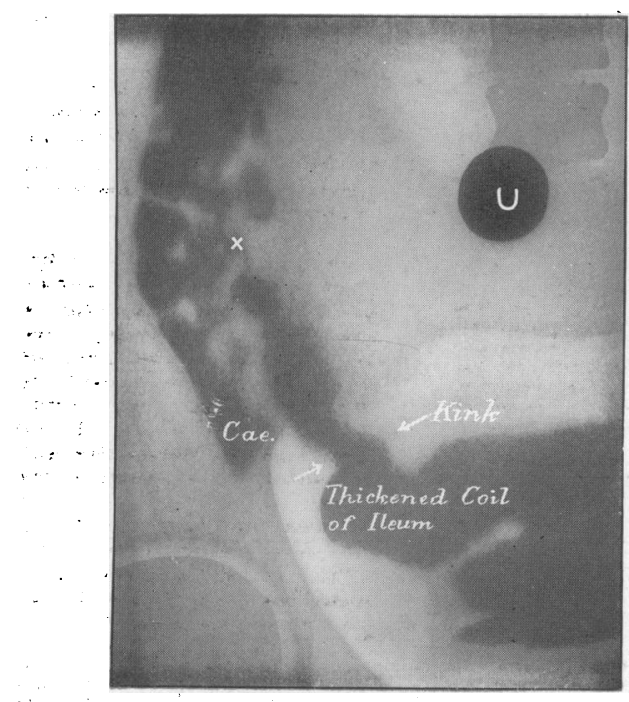

Fig. 16.

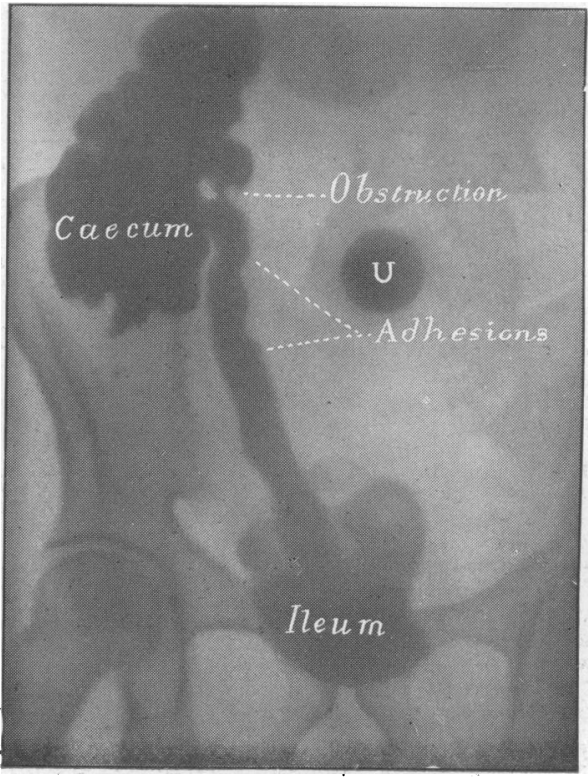

Fig. 17. 
jejunostomy, and the patient is relieved of pain, and is enabled, in many cases, to take full meals and to put on weight correspondingly, but the general symptoms and signs of intestinal stasis are not relieved, even though the immediate benefit may be süfficiently marked to satisfy the patient and all concerned. The staining of the skin remains; the poor circulation remains; the general depression remains or recurs before long; the nodular condition of the breasts is not rectified; headache, backache, and muscular pains often continue unabated; constipation and flatulence go on as before or return very soon.

The results of the operation of " short-circuiting" are very difierent, for all the signs and symptoms of intestinal stasis clear up at once.

In a case of duodenal ulcer the recumbency necessitated by the "shortcircuiting" is sufficiently prolonged to allow of the cure of the ulcer, and when the patients resume their ordinary mode of living the duodenal symptoms do not recur.

It is scarcely necessary to say that the secondary consequences of intestinal stasis may have been allowed to advance to a stage at which local treatment has become imperative. Thus there may be duocienal (or pyloric) ob. struction from the contraction of old cicatrized uleers (as in Figs. 5 and 17), or there may be one or more impacted gall stones (as in Fig. 12).

A most instructive case bearing on this point is the following:

A man of 56 had been in good health till two years ago, when he-began to have pain in the epigastrium pain was often relieved by vomiting The vomit by sisted of a pint of brown fluid : he never brought up red blood. The pain and romiting had been especially severe during the last six months, and he had lost

weight cluring these months.

On examination a succussion splash was observed over the stomach, but no other definite abnormalities were detected. The man, how ever, exhibited the typical signs of intestinal stasis

The $x$-ray examinations revenled a very definite train of changes. The stomach was greatly hypertrophied and clilated; very active peristaltic waves passed to the pylorus, but were unable to force more than traces of bismuth through the pyloris,

See a letter by Dr. F. P. Bremner in the Lancet, February 24th 1912 , D. 535, concerning bis own illness. A gastro-jejunostomy ha heerr performed by a very experienced surgeon but the benefit had pylorns but no improvement surgeon of equal eminence had closed the circuited "by Mr. Arbuthent had followed. Lastly, he was " shortof $x$-ray examinations after a bismuth meal, first, that thie gastro- this region being obviously the seat of fibrous stenosis. The diagnosis of stenosis was still further confirmed by the
occurrence of a series of strong antiperistaltic waves starting occurrence of a series of strong antiperistaltic waves starting
at the pylorus and working ulong the stomach toward the at the pylorus and working along the stomach toward the
fundus. This is a phenomenon rarely seen, and is considered to afford conclusive proof of pyloric stenosis, though the proof was perfect in the preoccurrence of antiperistalsis. After twenty-four hours more than half the bismuth was still in the stomach (Fig. 5A) there was a small collection of bismuth at the lower end terminal coil of the ileum, which rose as shown in Figs. 5 and $5 a$. The terminal coil was found to be firmly coil was found to be firmly men at a point 3 in. from its termination. This ileal kink was demonstrated to a number of medical men and students, who were able to satisfy themselves of its undoubted genuineness. On pressing with the hand upon the terminal coil the kinked point was found to be abparts immediately above and below the kink were freely movable. The result of manual pressure was to convert the angle of the link from an obtuse to an acute angle. In the upright posture the caecum fell, carrying down with it the upper $3 \mathrm{in}$. of the terminal coil; the portion of coil above the kink now hung down, forming a genuine sharp angle such as must inevitably have produced obstruction. No better illustration could be hadl of the fact that an ileal kink may only cause obstruction when the patient is upright. The appendix was shown at this and was shown at this and eight hours after the bismuth meal (Fig. 5A). It was placed immediately above and external to the kink, and it appeared normal. At this latter examination (fortyeight hours) there were stitl traces of bismuth in the stomach. There was considerable stasis in the large intestine, the greater part of intestine, the greater part of tained in the transverse colon at the end of seventy. two hours. A definite dia. gnosis was accordingly made to the effect that the patient pylorus or just beyond it, and pylorus or just beyond it, and ileal kink 3 in. from the end of the ileum, while the appendix was apparently

An operation was thereupon performed by Mr. upon performed by Mr. Arbuthnot Lane. As antici-
pated, the terminal coil of pated, the terminal coil of down at a point 3 in. from its end. The fibrous bands were so thick that Mr. Lane found it impracticable to divide them so as to release the coil. Accordingly he divided the ileum below the kink and sutured the divided end into sutured the divided end into the side of the rectum-his operation. The appendix lav close to the kinked portion of the ileum (see Fig. $5 \mathrm{~A}$ ). As enterostómy opening was functioning well, and secondly, that there was a well-marked ileal kink, an elongated rectum, and
other evidence of intestinal stasis. His general condition, too, had other evidence of intestinal stasis. His general condition, too, had
becn typical of intestinal stasis. He was restored to health, within a few weeks, and he has been carrying on a busy medical practice
uninterruptedly ever since July, 1911 (the operation was on May 22nd. 1911). 
expected, the appendix was perfectly normal and free from adhesions.

The pyloric region was next examined, and it was found that there was a cicatrizing duodenal ulcer just beyond the pylorus, producing obstruction. Mr. Lane therefore carried out a posterior gastro-jejunostomy for the relief of the obstriction.

The patient made a perfect recovery, and remains in good health.

No more perfect example could be desired of the relation of duodenal.ulcer to the ileal kink. Such a case ought to convince any one with an open mind. The presence of the ileal kink has been diagnosed by $\mathrm{Mr}$. Lane, after noting the dilated stomach, and recognizing the signs of intestinal stasis. The ray examinations were then carried out quite independently, without any information as to the clinical diagnosis already made by Mr. Lane; the result was a detailed and 'definite diagnosis, as stated already, expressed in clear language; leaving no loop-hole for escape. : The $x$-ray $\epsilon$ ridence was so clear that $I$ felt no loop-hole was needed. Lastly, there was the complete confirmation obtained at the operation, at which I was present.

In this case the diagnosis was made too late to save the duodenum ; permanent organic mischief had taken place and the only remedy was to exclude the duodenum from the circuit. Had this been done and nothing more, according to the usual practice, the relief would have been shortlived; the ileal kink would have asserted itself : with increase persistence, and a further operation would soon have been necessary for the relief of the intestinal stasis. Moreover, it is quite clear that the removal of the appendix at the time of performing the gastro-jejunostomy would have been of no advantage in this case, since it was healthy.

The moral is obvious: Make the diagnosis early, before the duodenal distension has proceeded to the stage of ulceration. I have shown how this may be done by $x$-ray examinations after a bismuth meal, and it is now open to all radiographers to supply definite and trustworthy information concerning the duodenum. The value of my method has been challenged by certain observers, who have not, however, attempted to apply it. All must agree that the value of any method of diagnosis is to be measured by its power of supplying trustworthy diagnoses. $\mathrm{My}$ method will bear the most severe test that can be applied : It enables the normal duodenum to be examined and to be reported "normal"; it enables all degrees of abnormality of size, shape, and activity to be ascertained and astual $x$-ray plotographs to be talsen. My results have been checked by competent surgeons at numerous operations, at many of which I have myself been present. The fluorescent screen phenomena of the duodenum have been observed with me by a number of physicians, surgeons, and students. In short, the subject is far beyond the experimental stage, and it has become the business of radiographers to devote the necessary time' and patience to the further study of the duodenum.

\section{Effect of the Upright Posture.}

The changes which lead to duodenal distension and to ileal obstruction from a kink are only brought into play in the upright posture. When the patient is put to bed the ileum and the jejunum return to their normal position, and the kinks are abolished for the time being except in severe cases. . This explains the fact that these patients are often quite comfortable while thoy are kept in bed, but the symptoms come on again when they get up. It also explains the fact that radiographic evidence of kinking, whether duodenal or ileal, is not always obtainable in patients who are kept in bed. Hence patients should be kept up, and on full diet as far as permissible, until the $x$-ray examinations are completed.

Duodenal Ulcer.

The fact that duodenal ulcer is a secondary condition dependent upon microbic infection arising in the lower tracts of the alimentary canal has been recognized of late by the well-known surgeon Mr. Moynilian, ${ }^{3}$ who has performed the operation of gastro-jejunostomy for duodenal ulcer in a very large number of eases. He has also acknowledged the drawbacks " of the operation, including the liability to the subsequent formation of a jejunal ulcer, or of a gastro-jejunal ulcer, at the new opening. Mr. Mayo. Robson, also a prominent supporter of the operation of gastro-jejunostomy for the trentment of - duodenal ulcer, has recently written a paper ${ }^{4}$ with the object of drawing attention to the same danger. These ulcers result from the microbic invasion of the small intestine, already shown to be due to the damming back of the intestinal contents at the lower end of the ileum.

The Appendix.

Mr. Moynihan, however, ${ }^{3}$ attributes the chief part in the formation of duodenal ulcer to the appendix, and he now removes this organ in ten out of every dozen operations of gastro-jejunostomy. He regards the appendix as the source of the septic infection, but the conditions he describes (and illustrates) are for the most part quiescent cicatricial or obliterative changes such as would certainly not act as foci of infection.

It is true, however, that the appendix is the primary cause of intestinal stasis in a particular group of cases. In this group (of which I have now seen a number of convincing examples) the proximal portion of the appendix is involved with the caecum in fibrous..bands in such a way. that the appendix actually controls, or even obstructs the end of the ileum while the patient is upright. In one case the most extreme of the kind I have seen as yet (Figs. 6 and $\left.6_{A}\right)$, the ileo-caecal junction was actually indented by the appendix, which was so fixed by a newly. formed fibrous band that it held up and obstructed the end of the ileum whenever the patient was in the uprigbt posture. The last four inches of the ileum were dilated, and obviously much hypertrophied. (There had been great tenderness to pressure in the right iliac fossa). In this case the removal of the appendix relieved all the obsisuction, and put an end to the stasis. A surgeon unfammiliar with this. condition would naturally attribute the cure of his patient to the removal of the appendix, whereas the result is due, in reality, to the relief of the stasis. Figs. 7 and $7 \mathrm{~A}$ represent a less extreme degree of a similar condition. In the less extreme cases the part played by the appendix in obstructing the outflow from the ileum when the patient is upright, is easily overlooked. In neither case (Figs. 6 or 7) were there any ileal adhesions.

The appendix is shown to contain bismuth in most cases during one or more of the examinations after a bismuth meal, and its position may be ascertained as a rule (Figs. 8 , $\left.9,10,12,13,14,{ }^{\prime} 15\right)$. Very often it lies concealed by the caecum, and a view of it is only to be obtained by displacing. the caecum by pressing with the hand in the riglit ilias; fossa (Fig. 5A). On removing the pressure the caecum often falls back into place and so hides the appendix, and. it is often impossible to obtain a photograph of the appendix although an accurate tracing may be made while the caecum is held out of the way (Fig. $5 \mathrm{~A}$ ). In certain cases the patient is himself able to keep up enough pressure with his right hand in the right iliac fossa to raise the caecum and enable a photograph of the appendix to be taken. The tip of the appendix frequently appears fixed, so that the appendix pivots round its tip during the movements of respiration, and, though the proximal part of the appendix is freely movable on pressure, the tip may be immobile (Fig. 12). This fixation of the tip is, as a rule, no evidence of discase. On inspecting the appendix at a subsequent operation the tip is seen to be fixed loosely in a bed of fat: (as in Fig. 12). An $x$-ray plotograph of the appendix may show: one or more sharp angles (as in Figs. 8 and 9), but pressure with the hand during the fluorescent screen examination will show that these angles are only apparent, being due to the circumstance that the appendix does not lie in the plane of the picture in the whole of its length.

In some cases the proximal or the middle portion of the appendix is found to be fixed by adhesions in such a way as to become kinked when the patient is upright (Fig. 7). In such cases it is usual to find that the distal portion of the appendix alone contains bismuth, that which entered tho proximal portion having been forced back into the caecum by the tension upon the appendix produced by the weight of the caecum (Fig. 7).

Tenderness to pressure over the appendix is certainly an important diagnostic point. The tenderness is sometimes localized accurately to the appendix, often to its proximal end (Figs. 14 and 15), and this tender point may be elicited at a number of consecutive examinations. In many other cases, where a tender point exists in the right 
iliac fossa and has been thought to arise from an inflamed appendix, careful manipulation during the $x$-ray examinations may show that the appendix itself is quite free from tenderness to pressure, while some portion of the terminal coil of the ileum is the seat of the tenderness. The usual points at which tenderness is found along the terminal coil of the ileum are three: (1) The point at which the terminal coil of the ileum crosses the pelvic brim ; (2) the ileo-caecal entrance; and (3) the seat of an ileal kink when one is present.

\section{The Ileal Kints.}

Mr. Moynihan stated recently ${ }^{5}$ that " he had very rarely found an ileal kink, and never without the plain evidence of an appendicitis, to which he believed the link to be secondary." As a matter of fact the typical ileal kink, of which I have now seen many examples (see Figs. 5, 5A, 11,16 , and 17), has no connexion whatever with the appendix ${ }^{16}$ unless quite accidentally, as in Fig. 15, ivhere the ileum and the appendix are kinked by the same band. I have described the ileal kink elsewhere, ${ }^{16}$ and there is no need to repest the description. The appendix is quite separate from the ileal end coil ; I have seen this clearly demonstrated at numerous operations, and I have given several instances in the present communication (Figs. 5, 11 13,16 , and 17).

The anatomical relations of the parts concerned in ileal kinking have been carefully described and beautifully illustrated by several American sürgeons, 78910 "who are now carrying out Mr. Lane's operation of "short-circuiting" for the curo of intestinal stasis with most satisfactory results.

Intestinal stasis forms one of the most important and far-reaching branches of radiographic research, and the systematic investigation of the alimentary tract by the $x$ rays after a bismuth meal is rapidly becoming an essential preliminary to intestinal surgery, and indeed to medical treatment of the digestive system.

REFERENCES.

1 Radiography in Intestinal stasis, Lancet, December 30th, 1911 , and Proc. Rny. Soc. Mel. December, 1911. 2 Duodenal Obstiction as Shown ly Radiography, Burrst Mepical JoonNat, May 20th, 1911: "Some Points in the Diagnosis and Treatment of Chronic Duodenal Ulcer, Lancet, January 6th, 1912 . 4 Jejunal and Gastro-Jejunal Ulcers, Symploms and Signs in some Abdominal Diseases, British Medić JotrNat, February 17th, 1912. "6 Radiographic, Demonstration of Lane's Ileal Kink, Practilioner, April, 1911. 'Tranklin H. Martin, Surgery, Gymaecology, and Obstetrics, January, 1911, p. 34.8 Chas. H. Mayo, ibid., March, 1911, 1). 227. 9 F. Gregory Connell, ibid., Norember, 1911, 1. 485. ${ }^{10}$ Geo. Tulley Vaughan, ibid., February, 1912. ${ }^{11}$ Chronic Intestinal Stasis. W. Arbuthnot Lane, Britisf Medical Jouriai May 4th, 1912, pp. 991-2. 12'R. Murray Leslie, Clinical Journti, May lst, 1912

\section{THE AUTOINOCULATION TEST IN TUBERCULOSIS.*}

\author{
BY H. WARREN CROWE, M.D.OxoN., \\ YELVERTON, DEVON.
}

Is 1906 Wright $^{1}$ described spontaneous fluctuations of the tuberculo-opsonic index in febrile cases of tuberculosis and affirmed the possibility of determining whether any given fever were associated with the tubercle bacillus, by observing a series of indices, and marking the presence or absence of thes fluctuations. The explanation Wright gave of this phenomenon seems quite convincing. He postulated a periodic change in the amount of toxic products entering the blood from a diseased focus. By day increased movement and bodily activity would, even when a patient was confined to bed, result in an increase of the blood flow throngh the whole organism, and toxic material would be washed out the more readily from the focus of disease into the general circulation. To this the system must respond by an increased production of immune substances. Hence come the fluctuations in the tuberculo-opsonic index, which, in point of fact, is an indicator of the amount of such response. To this periodic flow of toxic material Wright applied the term "spontaneous autoinoculation."

Freeman ${ }^{2}$ found that massage applied to a diseased joint was followed by an increased amount of toxin in the circulation, demonstrable again by a very definite

Read in opening a discussion at the Plymouth Medical Sociely on the diagnosis and treatment of tuberculosis. fluctuation in the opsonic index, provided only that the index was determined in respect of the causal organism. This he termed an artificial or induced autoinocnlation, and he was able to utilize his discovery to elucidate the nature of joint troubles of doubtful origin. The example he first quoted was that of a knee-joint said to be tuberculous; after massage the tuberculo-opsonic index did not fluctuate, whilst the index determined in respeet of the gonococcus gave a mirked and typical fluctuation. The inference drawn by Freeman was that the disease must bo gonorrhoea! and not tuberculous.

Hereupon Wright an 7 his collaborators, ${ }^{8}$ following out the same line of experimentation, showed that a very considerable number of agencies, such as exercise, ope:a tion, Bier's bandage, and even physical examination of the chest, were capable of inducing atutoinoculation.

Patterson elaboratel his treatment for phithisis, consisting of the scienti ic induction of autoinoculation by carefully graduated lakour, and in connexion therewitl Inman ${ }^{4}$ found that as long as disease was still present the varying grades of work produced fluctuations in the opsonic index, whilst in thosa apparently cured the most arduous labour was followed by no fluctuation whatever.

Lastly, I may refer to Inman's recent paper, ${ }^{5}$ in which he gives the result of 100 consecutive autoinoculation tests undertaken as a means of diagnosis in cases suspected of phthisis. These tests, however, suffer considerably from the fact that the exercise which was to excite an auto inoculation was frequently insufficient. : It is essential that in all tests undertaken for diagnosis this source of error should be rigorously excluded, and as our experience has grown it can now be: claimed that such is the case.

The apparatus which $I$ now use is a copy of the one suggested by Dr. Willcox for testing the lungs at St. Mary"s Hospital. By its moans forced respiration continued orer a period of some minutes can be used to induce auto inoculation in a tuberculous lung. The presence or absence of such antoinoculation is then determined by measuring the opsonic index before and at varying periods after the exercise.

Now there is a large number of diagnostic tests for tuberculosis, and it is difficult to decide which is the best unless one has some definite notion of the fumetion and characters which such a test should possess.

It would seem, therefore, to be of advantage to formu. late some standard whereby the merits of every test may be adjudicated. A little thought will give us certain canons to which an ideal test should conform. First, it must be harmless; secondly, it must confine itself to pointing ont the presence of active or smouldering tubercle -it must not be too penetrative; thirdly, the answer given be it must be unmistakable; fourthly, it must be universally applicable; fifthly, it must be simple ; sixthly. it should be practicable after treatment to determine the time when cure is complete; and, lastly, the ideal tost must be infallible. Of these saven canons, then, it will hardly be denied that the first and the last are the most important. Harmlessness and infallibility must be our watchwords in the search-the touchstone of our success.

In the series of 61 autoinoculation tests which form tho basis of this paper, I have excluded none that have been undertaken for phthisis during the last three years; 23 are crucial cases in which diagnosis of tuberculosis was to be definitely made or denied. In each case a definite answer was given, and in no case, so far, have I had any evidenco that the verdict was wroug. The following are examples:

Test 1.-A girl, apparently in quite an advanced stage of phthisis, was sent to Dartmoor by a London specialist with an extremely bad prognosis. In all good faith I treated her rigorously for lung disease. The course of the case, however, threw some doubt on the diagnosis, and it seemed advisable to take steps to decide the question. With a view to obtaining evidence as to the possibility of inducing autoinoculation, she was maide to walk for two hours. The opsonic index remained normal. The test was repented, with the same result. Thereupon further treatment was discontinued, and this somentrat hrsterical invalid was told to lead an ordinary life. No further symptoms have developed.

T'est 2.-A young woman, four months after an attack of influenza, had a slightly raised temperature at night, but showed no physical signs. She was made to walk two miles, and the index was determined of specimens of blood taken before, one hour after, six hours after, and again the next morning. The readings were: $0.85,1.15,0.7$, and 1.3, an immediate rise, followed by a fali, and then a second rise. This is a typical result whęre tubercle is present. I had no 